\title{
Deformation of multiwalled nanometer-sized carbon capsules
}

\author{
Koji Asaka ${ }^{a}$ \\ Special Research Project on Nanoscience, Graduate School of Pure and Applied Sciences, University of \\ Tsukuba, Tsukuba 305-8571, Japan \\ Ryoei Kato \\ Institute of Materials Science, Graduate School of Pure and Applied Sciences, University of Tsukuba, \\ Tsukuba 305-8573, Japan \\ Kun'ichi Miyazawa \\ Fuel Cell Materials Center, National Institute for Materials Science, 1-1, Namiki, Tsukuba 305-0044, Japan \\ Tokushi Kizuka \\ Institute of Materials Science, Graduate School of Pure and Applied Sciences, University of Tsukuba, \\ Tsukuba 305-8573, Japan and Special Research Project on Nanoscience, Graduate School of Pure and \\ Applied Sciences, University of Tsukuba, Tsukuba 305-8573, Japan and PREST, JST, Tsukuba 305- \\ 8573, Japan
}

(Received 14 June 2006; accepted 25 September 2006; published online 10 November 2006)

\begin{abstract}
The deformation of isolated multiwalled carbon capsules of a few nanometers in diameter was observed by in situ transmission electron microscopy with simultaneous force measurement by means of an optical cantilever method as used in atomic force microscopy. The mechanical properties of the carbon nanocapsules were investigated on an atomic scale. A carbon nanocapsule of $2.50 \pm 0.06 \mathrm{~nm}$ in diameter was selected and compressed upto a strain of 0.06 at a force of up to $4.5 \pm 0.5 \mathrm{nN}$ using a nanometer-sized gold tip of a microcantilever. By subsequent retraction, we measured the force between the carbon nanocapsule and the gold tip, and assessed the adhesion.

(C) 2006 American Institute of Physics. [DOI: 10.1063/1.2387872]
\end{abstract}

Since the discovery of fullerene $\mathrm{C}_{60}$ molecules, ${ }^{1}$ various nanometer-sized hollow cage structures of carbon and boronnitride have been synthesized. They are called giant fullerenes, ${ }^{2}$ single-walled ${ }^{3,4}$ and multiwalled nanotubules, 5,6 onions, ${ }^{7-9}$ spheres, ${ }^{10}$ cubes, ${ }^{11}$ hollow nanoparticles,,${ }^{9,12}$ cones, ${ }^{15,16}$ and horns. ${ }^{17}$ The mechanical properties originating from such peculiar structures have been studied both theoretically and experimentally. For a $\mathrm{C}_{60}$ molecule, the bulk modulus was predicted to be $\sim 700 \mathrm{GPa}$ by elastic continuum mechanics and calculations via the tight-binding method, ${ }^{18,19}$ and compressive and tensile deformations were investigated by molecular dynamics simulations and continuum mechanics. ${ }^{20-23}$ On the other hand, it was reported that $\mathrm{C}_{60}$ molecules were stable in collisions with silicon and graphite surfaces with an impact energy of up to $200 \mathrm{eV}$ in a vacuum chamber. ${ }^{24}$ We recently developed an experimental method to manipulate nanometer-sized carbon tips in a transmission electron microscope ${ }^{25,26}$ and synthesized a different type of fullerene $\mathrm{C}_{60}$, i.e., multiwalled nanometer-sized carbon capsules. ${ }^{27}$ In this letter, we demonstrate in situ atomistic observations and simultaneous force measurements of the deformation of individual carbon nanocapsules.

We synthesized single crystalline whiskers composed of $\mathrm{C}_{60}$ molecules with submicrometer diameters by a liquidliquid interfacial precipitation method using a toluene solution saturated with fullerene powders of $\mathrm{C}_{60}:-3 \mathrm{~mol} \%\left(\eta^{2}\right.$ $\left.-\mathrm{C}_{60}\right) \mathrm{Pt}\left(\mathrm{PPh}_{3}\right)_{2}$ and isopropyl alcohol. ${ }^{28,29}$ The crystalline $\mathrm{C}_{60}$ whiskers were heated in high vacuum at $1373 \mathrm{~K}$ for $30 \mathrm{~min}$ to transform them into amorphous carbon whiskers. The transformed whiskers were then dispersed on the tip of a

\footnotetext{
a) Author to whom correspondence should be addressed; electronic mail: asaka@sakura.cc.tsukuba.ac.jp
}

gold plate of $50 \mu \mathrm{m}$ thickness. The plate was mounted on a specimen holder on a transmission electron microscope equipped with an optical cantilever force measurement system. ${ }^{25}$ The nanometer-sized silicon tip of the cantilever, as used for atomic force microscopy, was coated with a gold film of 5-10 nm thickness, and was then fixed in front of a tube type piezoelectric element on another specimen holder. The cantilever tip was brought into contact with one of the amorphous carbon whiskers at every atomistic interval inside the combined microscope. We impressed a current into the whisker at room temperature in a vacuum of $1 \times 10^{-5} \mathrm{~Pa}$ to synthesize multiwalled carbon nanocapsules aggregating to the whisker. ${ }^{27}$ We separated the cantilever tip from the whisker and stopped current impression [Fig. 1(a)]. The transmission electron microscope was operated at an acceleration voltage of $200 \mathrm{kV}$. Under the observation using the combined microscope, we could discriminate each layer of the nanocapsules as a black line and measure distance until half-

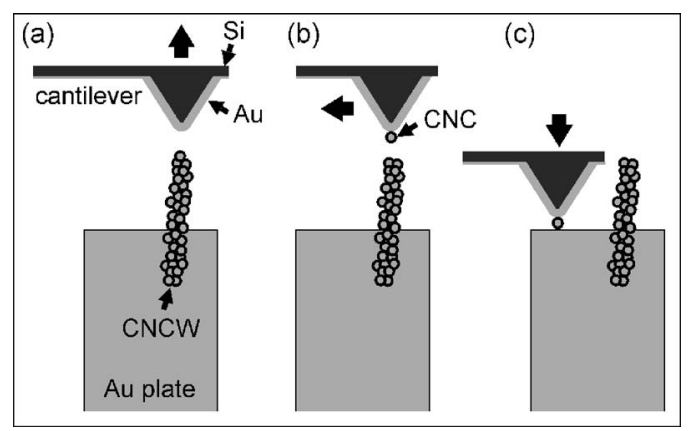

FIG. 1. Illustration of operation procedure. (a) Initial configuration of carbon nanocapsule whisker (CNCW), (b) transfer of multiwalled carbon nanocapsule (CNC) to surface of gold plate, and (c) deformation. 


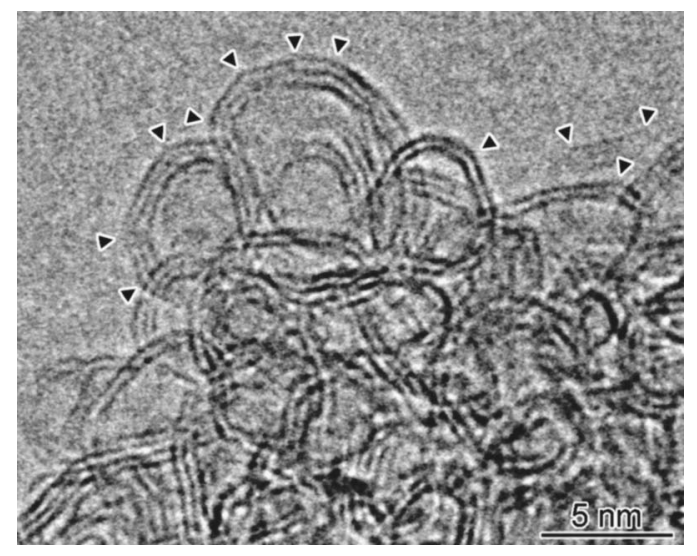

FIG. 2. High-resolution image of multiwalled carbon nanocapsules.

width of the line, i.e., $0.06 \mathrm{~nm}$. Thus, we analyzed the structural variation under the spatial resolution. The deformation dynamics was observed in situ using a television capture system. The time resolution of image observations was $17 \mathrm{~ms}$. The variations in force during deformation were measured by the optical lever method.

Figure 2 shows a high-resolution image of multiwalled carbon nanocapsules on a whisker surface. The diameter of the nanocapsules ranges from 3 to $10 \mathrm{~nm}$. The nanocapsules are composed of walls with two to four atomic layers. The spacing of the atomic layers is $0.34 \pm 0.06 \mathrm{~nm}$, corresponding to the interlayer spacing of graphite. Well-defined angles are frequently observed on the outer walls of the nanocapsules, as shown by the arrowheads in Fig. 2. We then picked one of the nanocapsules [Fig. 1(b)] and pressed it into the surface of the gold plate using the cantilever tip [Fig. 1(c)]. In the procedure shown in Fig. 1(c), the nanocapsule was supported by two gold surfaces of the cantilever tip and the plate. The strength of both gold/nanocapsule interfaces increases with their areas. Therefore, when the cantilever tip was pulled from the plate, separation occurred at the smaller interface. During the contact-separation procedure, the area between the cantilever tip and the nanocapsule became smaller and the nanocapsule transferred from the cantilever tip to the gold plate.

Figure 3 shows time-sequential high-resolution images of the deformation of the nanocapsule. The compression and subsequent retraction of the nanocapsule were repeated several times. The dark regions at the top and the bottom show the cantilever tip and the surface of the gold plate, respectively. The bright regions indicate vacuum. Figure 4 shows the relationship between the cantilever tip displacement and the force during the deformation seen in Fig. 3. The points indicated by arrowheads $\mathrm{a}-\mathrm{h}$ in Fig. 4 correspond to the times at which Figs. 3(a)-3(h) were observed. In Fig. 3(a), the width of the nanocapsule along the compressing direction is $2.50 \pm 0.06 \mathrm{~nm}$, and the walls are two atomic layers. When the distance between the cantilever tip and the nanocapsule is reduced to $1.0 \mathrm{~nm}$, the cantilever tip jumps into contact with the surface of the nanocapsule, as seen in Fig. 3(b). The attractive force at the jump is found to be $2.0 \mathrm{nN}$ at b in Fig. 4. We shift the cantilever tip at a speed of $2.1 \mathrm{~nm} / \mathrm{s}$ and compress the nanocapsule along the direction indicated by the bigger arrow in Fig. 3(b). The force increases from 0 [Figs. 3(c) and 4(c)] to 4.5 $\pm 0.5 \mathrm{nN}$ [Figs. 3(e) and 4(e)], and the nanocapsule is collapsed by $0.14 \pm 0.06 \mathrm{~nm}$, correspondDownloaded 09 Nov 2009 to 130.158.56.186. Redistribution subject

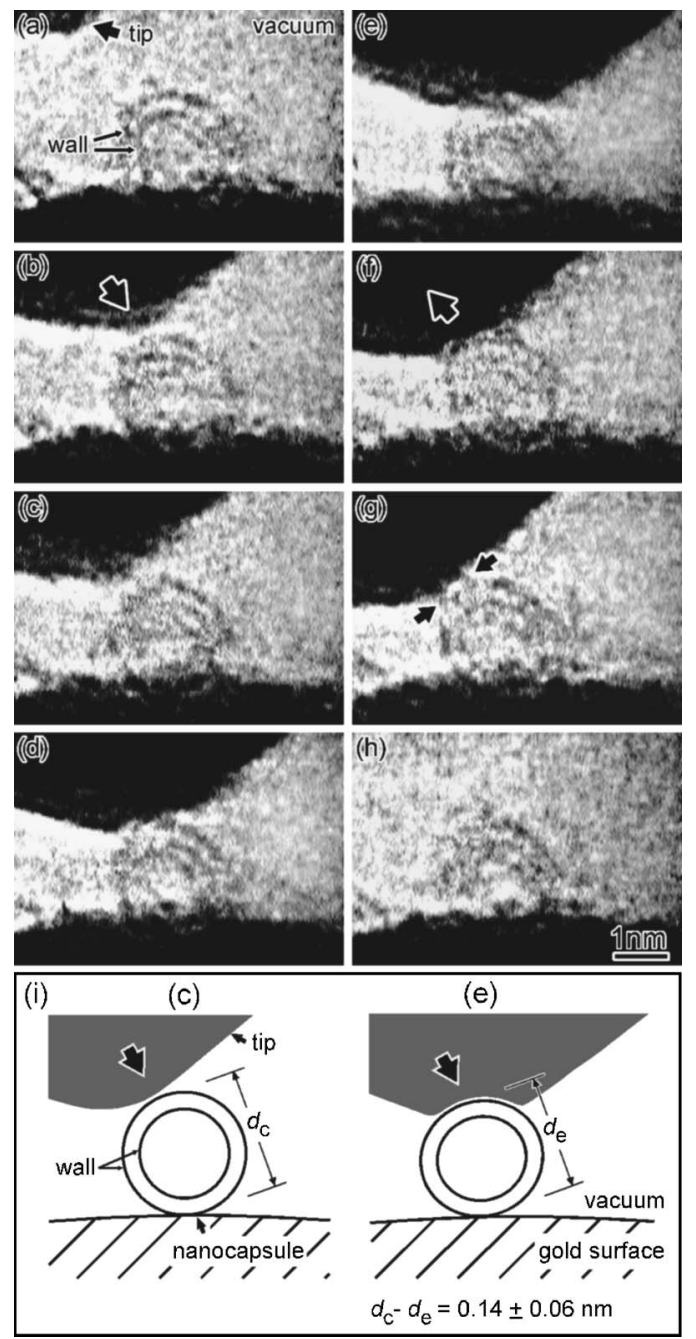

FIG. 3. (a)-(h) Time-sequential images of deformation of multiwalled carbon nanocapsule. The diameter of the nanocapsule in (a) is $2.50 \pm 0.06 \mathrm{~nm}$. (i) Illustration of deformation in (c) and (e).

ing to a strain of 0.06. The deformation in Figs. 3(c) and 3(e) is illustrated in Fig. 3(i). During the compression in Figs. $3(\mathrm{~d})$ and 3(e), the cantilever tip is dented at the interface between the tip and nanocapsule, showing that the nanocapsule is harder than the gold surface. The force increases up to $5.5 \pm 0.5 \mathrm{nN}$, at which point the cantilever tip is retracted along the direction indicated by the arrow in Fig. 3(f). Dur-

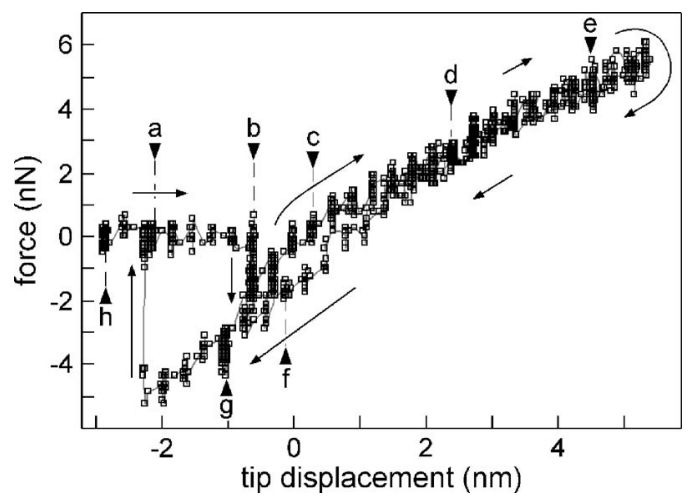

FIG. 4. Displacement-force curve during deformation in Fig. 3. The times indicated by arrowheads $\mathrm{a}-\mathrm{h}$ in Fig. 3 represent the times at which the images in Figs. 3(a)-3(h) were observed. At b the cantilever tip jumps into contact with the nanocapsule.

to AIP license or copyright; see http://apl.aip.org/apl/copyright.jsp 
ing the retraction, the cantilever tip pulls carbon atoms in the outermost wall and adheres to the nanocapsule, as indicated by the two smaller arrows in Fig. 3(g). Finally, the cantilever tip completely separates from the nanocapsule at a distance of $0.20 \pm 0.06 \mathrm{~nm}$ and a force of $4.9 \pm 0.5 \mathrm{nN}$, as shown in Figs. 3(h) and 4(h).

The compressive force is proportional to the displacement from c to e in Fig. 4; the nanocapsule elastically deforms in this region. The spring constant of the nanocapsule is estimated to be $40 \pm 20 \mathrm{~N} / \mathrm{m}$. Based on the relationship between the force $(F)$ and distance $(x)$, the elastic energy required to compress the nanocapsule up to a strain of 0.06 is roughly calculated to be $\frac{1}{2} F x \sim 2.0 \mathrm{eV}$. According to the simulation of compressive deformation of $\mathrm{C}_{60}, \mathrm{C}_{80}$, or $\mathrm{C}_{180}$, the value $\sim 2 \mathrm{eV}$ is on the same order as the elastic energy. ${ }^{24}$

In the same way as the calculation for the elastic energy during the compression, the energy required to separate the cantilever tip from the nanocapsule during the retraction is estimated. The force and distance at the separation are $4.9 \pm 0.5 \mathrm{nN}$ and $0.2 \pm 0.06 \mathrm{~nm}$; the bonding energy is calculated to be $\sim 3.1 \mathrm{eV}$ in total. The total energy is larger than the cohesive energy of $\mathrm{C}_{60}$ crystals by van der Waals forces, i.e., $1.5 \sim 2 \mathrm{eV},{ }^{30,31}$ When we assumed that the interface shape of the present nanocapsule is circular, the area is estimated to be $0.25 \mathrm{~nm}^{2}$ from the diameter and the bonding energy per atom is calculated to be $\sim 0.31 \mathrm{eV}$. The energy per atom is similar to the adsorption energy of a gold atom on graphite, i.e., 0.45 , and $0.64 \mathrm{eV} .^{32,33}$ It was also reported that a strong interaction occurred for $\mathrm{C}_{60}$ molecules absorbed on gold surfaces. ${ }^{34,35}$ Several studies indicated that the $\mathrm{C}_{60}$-gold interaction was due to the charge transfer and chemisorption. ${ }^{36-39}$ It is suggested in this study that similar interaction occurs at the interface between the gold tip and the nanocapsule.

In conclusion, we investigated the compression of individual multiwalled carbon nanocapsules. The displacementforce curve was obtained by in situ transmission electron microscopy, and the spring constant and the adhesion force with the gold surface were estimated.

This study was partly supported by the funds for the Special Research Project on Nanoscience and the University Research projects of the University of Tsukuba, and a Grantin-Aid for Scientific Research from the Ministry of Education, Culture, Sports, Science and Technology in Japan.

${ }^{1}$ H. W. Kroto, J. R. Heath, S. C. O’Brien, R. F. Curl, and R. E. Smalley, Nature (London) 318, 162 (1985).

${ }^{2}$ L. D. Lamb, D. R. Huffman, R. K. Workman, S. Howells, T. Chen, D. Sarid, and R. F. Ziolo, Science 255, 1413 (1992).

${ }^{3}$ S. Iijima and T. Ichihashi, Nature (London) 363, 603 (1993).

${ }^{4}$ D. S. Bethune, C. H. Kiang, M. S. de Vries, G. Gorman, R. Savoy, J.
Vazquez, and R. Beyers, Nature (London) 363, 605 (1993).

${ }^{5}$ S. Iijima, Nature (London) 354, 56 (1991).

${ }^{6}$ G. N. Chopra, R. J. Luyken, K. Cherrey, V. H. Crespi, M. L. Cohen, S. G. Louie, and A. Zettl, Science 269, 966 (1995).

${ }^{7}$ S. Iijima, J. Cryst. Growth 50, 675 (1980).

${ }^{8}$ O. Stéphan, Y. Bando, A. Loiseau, F. Willaime, N. Shramchenko, T. Tamiya, and T. Sato, Appl. Phys. A: Mater. Sci. Process. 67, 107 (1998).

${ }^{9}$ T. Oku, M. Kuno, H. Kitahara, and I. Narita, Int. J. Inorg. Mater. 3, 597 (2001).

${ }^{10}$ D. Ugarte, Nature (London) 359, 707 (1992).

${ }^{11}$ Y. Saito and T. Matsumoto, Nature (London) 392, 237 (1998).

${ }^{12}$ W. A. de Heer and D. Ugarte, Chem. Phys. Lett. 207, 480 (1993).

${ }^{13}$ R. S. Ruoff, D. C. Lorents, B. Chan, R. Malhotra, and S. Subramoney, Science 259, 346 (1993).

${ }^{14}$ M. Tomita, Y. Saito, and T. Hayashi, Jpn. J. Appl. Phys., Part 2 32, L280 (1993).

${ }^{15}$ K. Sattler, Carbon 33, 915 (1995).

${ }^{16}$ A. Krishnan, E. Dujardin, M. M. J. Treacy, J. Hugdahl, S. Lynum, and T. W. Ebbesen, Nature (London) 388, 451 (1997).

${ }^{17}$ S. Iijima, M. Yudasaka, R. Yamada, S. Bandow, K. Suenaga, F. Kokai, and K. Takahashi, Chem. Phys. Lett. 309, 165 (1999).

${ }^{18}$ R. S. Ruoff and A. L. Ruoff, Nature (London) 350, 663 (1991).

${ }^{19}$ S. J. Woo, S. H. Lee, E. Kim, K. H. Lee, Y. H. Lee, S. Y. Hwang, and I. C. Jeon, Phys. Lett. A 162, 501 (1992).

${ }^{20}$ D. W. Brenner, J. A. Harrison, C. T. White, and R. J. Colton, Thin Solid Films 206, 220 (1991).

${ }^{21}$ Z. X. Zhang, Z. Y. Pan, Y. X. Wang, Z. J. Li, and Q. Wei, Mod. Phys. Lett. B 17, 877 (2003).

${ }^{22}$ J. W. Kang and H. J. Hwang, Nanotechnology 15, 614 (2004).

${ }^{23}$ T. Xiao and K. Liao, Nanotechnology 14, 1197 (2003).

${ }^{24}$ R. D. Beck, P. S. John, M. M. Alvarez, F. Diederich, and R. L. Whetten, J. Phys. Chem. 95, 8402 (1991).

${ }^{25}$ T. Kizuka, H. Ohmi, T. Sumi, K. Kumazawa, S. Deguchi, M. Naruse, S. Fujisawa, S. Sasaki, A. Yabe, and Y. Enomoto, Jpn. J. Appl. Phys., Part 2 40, L170 (2001).

${ }^{26}$ K. Asaka and T. Kizuka, Phys. Rev. B 72, 115431 (2005).

${ }^{27}$ K. Asaka, R. Kato, Y. Maezono, R. Yoshizaki, K. Miyazawa, and T. Kizuka, Appl. Phys. Lett. 88, 051914 (2006).

${ }^{28}$ K. Miyazawa, A. Obayashi, and M. Kuwabara, J. Am. Ceram. Soc. 84, 3037 (2001).

${ }^{29}$ K. Miyazawa, J. Minato, H. Zhou, T. Taguchi, I. Honma, and T. Suga, J. Eur. Ceram. Soc. 26, 429 (2006).

${ }^{30} \mathrm{Ph}$. Lambin, A. A. Lucas, and J.-P. Vigneron, Phys. Rev. B 46, 1794 (1992).

${ }^{31}$ R. Luthi, E. Meyer, H. Haefke, L. Howald, W. Gutmannsbauer, and H.-J. Guntherodt, Science 266, 1979 (1994).

${ }^{32}$ P. Jensen, X. Blase, and P. Ordejon, Surf. Sci. 564, 173 (2004).

${ }^{33}$ R. Anton and I. Schneidereit, Phys. Rev. B 58, 13874 (1998).

${ }^{34}$ E. I. Altman and R. J. Colton, Surf. Sci. 279, 49 (1992).

${ }^{35}$ S. Howells, T. Chen, M. Gallagher, D. Sarid, D. L. Lichtenberger, L. L. Wright, C. D. Ray, D. R. Huffman, and L. D. Lamb, Surf. Sci. 274, 141 (1992).

${ }^{36}$ T. R. Ohno, Y. Chen, S. E. Harvey, G. H. Kroll, J. H. Weaver, R. E. Haufler, and R. E. Smalley, Phys. Rev. B 44, 13747 (1991).

${ }^{37}$ S. J. Chase, W. S. Bacsa, M. G. Mitch, L. J. Philione, and J. S. Lannin, Phys. Rev. B 46, 7873 (1992).

${ }^{38}$ A. J. Maxwell, P. A. Brühwiler, A. Nilsson, N. Mårtensson, and P. Rudolf, Phys. Rev. B 49, 10717 (1994).

${ }^{39}$ C.-T. Tzeng, W.-S. Lo, J.-Y. Yuh, R.-Y. Chu, and K.-D. Tsuei, Phys. Rev. B 61, 2263 (2000). 